\title{
Die „Heliosschule - Inklusive Universitätsschule der Stadt Köln“
}

\section{Zusammenfassung}

Die „Heliosschule - Inklusive Universitätsschule der Stadt Köln“ wurde 2015 in Betrieb genommen. Diese öffentliche Schule umfasst die Klassenstufen 1 bis 13 für etwa 1.100 Schülerinnen. Die Schule soll ein Modell für eine demokratische Schule auf dem Weg in die Inklusion in der Gegenwart sein, wobei Prinzipien der Beteiligung und Diversität wesentlich für das Modell sind. Der Text besteht aus drei Teilen: Erstens werden zentrale Elemente und Prinzipien der Schulgründung in kurzer Form dargestellt. Zweitens wird eine Zusammenfassung des inklusiven Vorgehens anhand der 10 Bausteine der inklusiven Didaktik gegeben. Abschließend werden einige Hindernisse auf dem Weg dieser inklusiven Schule genannt.

Schlüsselwörter: Schulentwicklung, Inklusion, Didaktik, Lernlandschaft, Selbstlernmethoden, Teamschule, rhythmisierter Ganztag, Projekte, Werkstätten

\section{The "Helios School - Inclusive University School of Cologne" Summary}

The "Heliosschool - Inclusive University School of Cologne" was established in 2015. This public school provides classes 1 to 13 for about 1,100 students. The school intends to be a contemporary model of democratic school in the way to inclusion, based on principles of participation and diversity, under the complex life conditions of today. The text contains three components: First, I will reconstruct some elements and principles that are fundamental for the foundation of this school. Second, I will give a comprehensive account of the inclusive concept of the Cologne school and the ways it works out in practice. I will elaborate on the pedagogical program of the school formulated in ten components of inclusive learning and teaching. Finally, I will discuss some main obstacles that we have encountered and had to overcome in establishing the school in Cologne.

Keywords: school development, inclusion, didactics, learning environment, independentlearning techniques, team school, rhythmitized all-day schooling, projects, workshops 
Die „Heliosschule“ wurde nach langen Jahren der konzeptionellen Vorbereitung 2015 von der Stadt Köln als öffentliche Schule gegründet. Ein gemeinsamer Vertrag mit der Universität zu Köln unter Genehmigung der Bezirksregierung sichert eine Nutzung für die Lehramtsausbildung, so dass eine universitär begleitete Praxisschule entsteht. Sie besteht aus einer zweizügigen Grundschule und einer vierzügigen Gesamtschule, wobei die Grundschüler*innen alle in die Sekundarstufe I übernommen werden können. Das Konzept der Schule ist ausführlich beschrieben (Reich, Asselhoven \& Kargl, 2015; Reich, 2018), und die Schule wird durch die Universität wissenschaftlich begleitet. Wichtige inhaltliche Orientierungen werden in Reich $(2012,2014,2017)$ dargestellt. Wesentlich ist bei der Konzeption, dass die Schule sowohl für eine exzellente inklusive Lehramtsausbildung stehen und hier neue Formen experimentell erproben soll als auch insbesondere Forschungen im Feld des Lernens, hier insbesondere in der inklusiven Erziehung und Bildung, und der Verbesserung der Qualität der Lehramtsausbildung ermöglichen will.

\section{Eckdaten der Schule}

Die Inklusive Universitätsschule ist nach dem Schulgelände „Heliosschule“ benannt. Auf diesem stand früher eine Fabrik, die unter anderem Leuchttürme produzierte. Ein Leuchtturm, früher zu Werbungszwecken genutzt, steht noch auf dem Gelände, was als schöne Metapher für diese Schule genommen werden kann. Wenn sie als Neubau etwa 2022/23 fertiggestellt sein wird, dann wird sie ca. 1.100 Schüler*innen haben, die die Primarstufe und die Sekundarstufen I und II besuchen. Zwischenzeitlich operiert die Schule an Übergangsstandorten. Der Neubau - das Modell wird näher in Kricke et al. (Kricke, Schanz, Reich \& Schneider, 2018) beschrieben - löst das herkömmliche Klassensystem in Lernlandschaften von je vier Klassen auf, wobei in den unteren Jahrgängen jahrgangsübergreifend gearbeitet wird. Die ca. 108 Schüler ${ }^{\star}$ innen pro Lernlandschaft der Stufen 1 bis 13 werden in sechs Stammgruppen unterteilt, die in den differenzierten Zonierungen der Landschaft lernen können. In der Lernlandschaft finden wir eine kleine Schule in einer großen, denn hier sind alle notwendigen Aspekte integriert: Garderobe, Ankommensbereich, Teamstation der Lehrkräfte und des multi-professionellen Teams, Küchenzeile, Waschräume und Toiletten, unterschiedliche Lernzonen einschließlich eines großen Bewegungsund Präsentationsraumes, den sich zwei Lernlandschaften teilen, Rückzugs- und Besprechungsräume. In den Teams mit eigener Teamstation sind alle Lehrkräfte und weiteres Personal aus dem Ganztag und der Schulsozialarbeit, Honorarkräfte, Studierende usw. integriert, so dass pro Lernlandschaft ca. 20 Personen und pro Stammgruppe von ca. 17 Schüler*innen mindestens zwei Lehrkräfte verantwortlich sind. Jeder Lehrkraft ist dabei ein Studierender im Praxissemester zugeordnet. Es gibt eine ganztägige Anwesenheit am Arbeitsplatz, der in der Teamstation untergebracht und mit moderner Digitalisierung - wie die gesamte Schule - ausgestattet ist. 
Zusätzlich gibt es eine Mensa, die mit einer Aula mit großer Bühne verbunden werden kann, Fachräume, die in den Naturwissenschaften ebenfalls als Lernlandschaft ausgelegt sind, ein Pflegebad, auch Räume für Schüler*innenfirmen, einen Sport-, Kunst- und Musikbereich sowie eine Lehrküche. Ein Verwaltungstrakt und Räume der Universität, die auch von der Schule mitgenutzt werden können, runden das Raumspektrum ab. Alle Lernlandschaften haben eigene Zugänge nach draußen über Terrassen, aber es steht durch die innerstädtische Lage nur ein recht kleiner Schulhof bereit.

Ähnlich wie bei der Laborschule Bielefeld gilt auch in Köln John Deweys „Laboratory School“ als Vorbild des Konzeptes. In seinem Buch The School and Society (Dewey, MW 1, S. 1-109), das zuerst 1900 publiziert wurde, beschrieb Dewey die Universitätsschule von Chicago, die 1896 an den Start ging und bis heute existiert, nach Modell und Durchführung. Diese Schule stellte damals eine grundlegend neue Form des Lernens dar, die insbesondere die Idee einer demokratischen Beteiligung der Lernenden als „Demokratie im Kleinen“ mit einem radikal veränderten Schultag unter Auflösung der Klassenzimmer und herkömmlichen Lehrpläne vorsah. Bereits für Dewey bot das überwiegende Instruktionslernen zu wenig Möglichkeiten, damit Lernende aktiv Wissen mit eigenen Problemstellungen und Erfahrungen erwerben, an sozialen Interaktionsprozessen teilnehmen und ihr eigenes Lernen selbstorganisiert und auch stärker praxisbezogen und lebensrelevant gestalten können. Mit seiner pragmatistischen Lerntheorie, die von einem emotionalen Ankerpunkt und Problemen ausgeht, um Lernvorgänge hinreichend motiviert zu eröffnen, setzte Dewey für die spätere Entwicklung von Lerntheorien bis zu heutigen konstruktivistischen Ansätzen entscheidende Impulse (vgl. Garrisson, Neubert \& Reich, 2012, 2016). Die Schule wurde von Dewey als eine Art miniature community oder embryonic society aufgefasst, was er später in Democracy and Education (Dewey, MW 9) ausführlich in einen Zusammenhang mit der notwendigen weiteren Demokratieentwicklung der Gesellschaft brachte. Auch das Konzept der „Heliosschule“ ist von der Idee geleitet, dass eine demokratische Schule die Entwicklung der Demokratie im Großen mitgestaltet. Gemeinsamkeiten mit Deweys Modell werden in Reich (2018) dargestellt.

Über 70 Akteure aus der Kölner Region waren an der ersten Entwicklung des Konzeptes beteiligt, darunter Studierende und Lehrkräfte aller Schulformen, Lehrende an der Universität aus unterschiedlichen Fachwissenschaften, Eltern, insbesondere aus dem Bereich der Inklusion, Akteure in der Schulsozialarbeit und Schulpsychologie, Schülerinnen und andere. Das Dewey-Center Köln bildete einen guten Hintergrund, und teacher training schools weltweit, insbesondere aus Finnland, konnten als Vorbilder herangezogen werden. Das Schulgelände wurde durch eine Bürgerbeteiligung, die sich gegen die Errichtung einer Shopping Mall wehrte, in einem stadtbekannten Beteiligungsverfahren gegen kommerzielle Interessen erstritten. Dies schafft gute Voraussetzungen, die Schule als gewolltes Projekt der Bürger ${ }^{\star}$ innen im Stadtteil Ehrenfeld zu bauen. Die Universität beauftragte mich und mein Team mit 
der wissenschaftlichen Leitung in der Gründungsphase und nach der Gründung; seit 2017 hat meine Nachfolgerin Lisa Rosen die Leitung für die universitäre Seite übernommen. Die Schulleitungen der Grundschule und der Gesamtschule sind Marion Hensel bzw. Andreas Niessen. Es gibt eine gemeinsame Lenkungsgruppe der Stadt als Schulträger, der Universität und der Bezirksregierung, in der Spitzen der jeweiligen Institutionen vertreten sind.

Die „Heliosschule“ ist nicht nur ein neuartiges Beispiel für eine von der Universität begleitete Ausbildungs- und Forschungsschule im Rahmen der Lehramtsausbildung in NRW, sondern sie ist auch ein Leuchtturmmodell in der Inklusion. Für den Neubau wird hierbei nicht nur Barrierefreiheit durch- und umgesetzt, sondern auch ein Modell von Lernlandschaften verwirklicht, das nachhaltig den Bedarfen und Bedürfnissen von heterogenen Lerngruppen in der Inklusion entsprechen kann (vgl. Montag Stiftungen, 2017; Kricke et al., 2018). Entscheidend bei der Konzipierung der Schule ist es, dass es zwar ein Rahmenkonzept für das Vorhaben gibt, dieses jedoch für die innere Schulentwicklung durch das Schulteam hinreichend Raum zur weiteren Entwicklung lässt. Schule wird im Konzept als ein sich wandelndes, lebendiges System verstanden, das alle beteiligten Akteure in die Mitgestaltung aktiv als Team einbeziehen muss (Kricke \& Reich, 2016).

Zwei grundlegende Schwierigkeiten mussten während des Gründungsprozesses bewältigt werden:

(1) Zunächst ist das traditionelle deutsche Schulsystem mit seinen unterschiedlichen Schulformen, der frühen Selektionsschranke nach der vierten Klasse in NRW, der zwar steigenden Inklusionsquote an Regelschulen, aber der gleichzeitigen steigenden Anzahl an Schüler*innen mit Förderbedarf, im Verhältnis zu den einheitlicheren Schulsystemen weltweit ein großer Nachteil, um tatsächlich heterogene Lerngruppen in hinreichender Breite an Regelschulen zu bilden. Das Konzept der „Heliosschule“ sieht deshalb ganz klar vor, solche Heterogenität repräsentativ für die Bevölkerung der Stadt Köln herzustellen, aber dies weist etliche Hürden im Schulrecht und in den geltenden Aufnahmeprozeduren der Schüler*innen auf. Zudem sieht das Konzept der Schule eine Schule von Klasse 1 bis 13 vor, was nur dadurch realisiert werden konnte, dass durch eine Umstellung von zweizügig auf vierzügig entsprechend der rechtlichen Vorgaben ein Übergang für alle Kinder gewährleistet werden konnte. Dies liegt daran, dass das Aufnahmeverfahren in die Gesamtschule nicht durch die Grundschule vorbestimmt sein darf. Das Schulministerium unter Frau Löhrmann lehnte einen Sonderfall einer Schule mit den Klassen 1 bis 13 ab, so dass zwei Schulen gegründet wurden, die unter dem Dach eines Neubaus stehen und als getrennte Schulen zwar äußerlich kaum wahrgenommen werden können, aber dem Dogma einer wenig flexiblen Schulpolitik, keine Leuchttürme der Veränderung zu schaffen, entsprechen. Dennoch gab und gibt es auf allen Ebenen auch in den zuständigen Behörden sehr viel Unterstützung für das Reformmodell. 
(2) Die traditionelle Lehramtsausbildung, die Theorie und Praxis nach wie vor stark trennt, ist die andere Schwierigkeit. Erst ein neues Lehrerausbildungsgesetz, das ein Praxissemester in NRW in der Masterphase einführte, ließ überhaupt die Möglichkeit zu, Studierende in einer längeren Praxisphase während des Studiums zu begleiten. Hier musste dann noch eine Sondergenehmigung erwirkt werden, um die maximale Zahl von fünf Studierenden pro Schule im Praxissemester zu erweitern. Pro Lehrkraft steht an der „Heliosschule“ ein Studierender in der Praxisphase zur Verfügung. Dies bedeutet, im Endausbau über 80 Studierende als Begleitpersonal zugleich auszubilden und praktisch einzusetzen.

Vor diesem Hintergrund ist die „Heliosschule“ eine öffentliche Schule, die durch die Universität in dreierlei Hinsicht begleitet und unterstützt wird:

1. Die Schule ist eine lehramtsausbildende Schule für die Praxisphasen aller Studierenden. Dabei werden die Studierenden, die ihr Praxissemester an der Schule verbringen, zuvor gezielt in Assessments ausgewählt und durch eine eigene inklusive Profilierung ausgebildet. Pro Jahr werden zunächst ca. 160 Studierende so ausgebildet. Diese inklusive Profilierung soll der Universität helfen, inklusive Studienanteile in der Ausbildung im Bachelor und Master zu erproben, bevor sie für die gesamte Lehramtsausbildung umgesetzt werden. Für Studierende entstehen Vorteile bei der späteren Bewerbung um eine Stelle. Im inklusiven Profil kommt es zu gemischten Ausbildungsteams sowohl zwischen den Bildungswissenschaften und den sonderpädagogischen Fachrichtungen als auch den inklusiven Fachdidaktiken. Lehrkräfte aus der Schule werden zusätzlich in die universitäre Ausbildung eingebunden, um eine bessere Verzahnung von Theorie und Praxis zu erreichen. Die Grundidee lautet: Wenn Inklusion an den Regelschulen besser gelingen soll, dann müssen auch die Hochschulen deutlich inklusiver vorgehen. Im Neubau der Schule investiert die Universiät aus eigenen Geldern freiwillig 4,5 Millionen für Räume und Ausstattung, damit die ca. 80 Studierenden an der Schule auch von der Universität angemessen vor Ort betreut werden können. Entsprechende Personalstellen sichern dieses Konzept ab.

2. Die Universität unterstützt die inklusive Schulentwicklung durch Forschungen, die gemeinsam zwischen der Schule und der Universität abgestimmt werden. Forschungsfragen werden so direkt mit der Schulpraxis verknüpft und sind nicht ausschließlich an die Interessen der Forscher*innen gebunden. Hierbei hat eine langfristige Forschung den Vorzug vor kurzfristigen Untersuchungen.

3. Gemeinsame Projekte zwischen Schule und Universität sollen zusätzlich sowohl die Entwicklungsseite der Schule als auch die Forschungsseite, insbesondere durch Einbezug der internationalen Kontakte und Netzwerke sowohl der Schule als auch der Universität, verbessern helfen. Hierbei können auch Austauschprogramme für das Personal hilfreich sein. 
Die „Heliosschule - Inklusive Universitätsschule der Stadt Köln“ |

\section{Das Konzept der Schule}

Das Leitbild der Schule (vgl. Reich et al., 2015) baut auf den 10 Bausteinen der inklusiven Didaktik nach Reich (2014) auf. Wesentliche Punkte dieses Konzepts werden hier in knapper Form zusammengefasst:

\begin{tabular}{|c|c|c|}
\hline $\begin{array}{c}\text { Merk- } \\
\text { mal }\end{array}$ & Leitprinzipien & Beispiele konkreter Maßnahmen \\
\hline 1 & $\begin{array}{l}\text { Eine inklusive Lernkul- } \\
\text { tur mit Blick auf positive } \\
\text { wechselseitige Bezie- } \\
\text { hungen in stützender } \\
\text { Struktur, mit Gerechtig- } \\
\text { keit und Fürsorge, die } \\
\text { auf Diversität, } \\
\text { Kooperation und Team- } \\
\text { teaching in multiprofes- } \\
\text { sionellen Teams baut. }\end{array}$ & $\begin{array}{l}\text { - Das inklusive Leitbild wird von allen, die an der Schule beteiligt } \\
\text { sind, geteilt und nach außen vertreten. } \\
\text { - Eine gute Beziehungskultur auf allen Ebenen wird von allen ange- } \\
\text { strebt. } \\
\text { - Multiprofessionelle Teams schließen Lehrkräfte, Sonderschullehr- } \\
\text { kräfte, Ganztagskräfte, Kräfte aus der Schulsozialarbeit, Schulpsy- } \\
\text { chologie, Therapie, Studierende im Praktikum und andere mit ein. } \\
\text { - Das multiprofessionelle Team versteht sich als ein lernendes Team, } \\
\text { das Fort- und Weiterbildungen kontinuierlich wahrnimmt und die } \\
\text { wissenschaftliche Forschung aktiv unterstützt. } \\
\text { - In Lernlandschaften werden intensive und vielfältige Interaktionen } \\
\text { zwischen allen Lehrenden und Lernenden so begleitet, dass ein } \\
\text { kontinuierliches Wachstum für alle möglich ist. } \\
\text { - Peer-to-peer-Lernen wird in allen Formen unterstützt. } \\
\text { - Alle sind ganztägig gemeinsam anwesend. }\end{array}$ \\
\hline 2 & $\begin{array}{l}\text { Die Schule verfolgt } \\
\text { Prinzipien demokrati- } \\
\text { scher Beteiligung und } \\
\text { eine Verbesserung } \\
\text { möglichst gerechter } \\
\text { Chancen in einer be- } \\
\text { wusst heterogenen und } \\
\text { dabei für die Stadt Köln } \\
\text { repräsentativ gebildeten } \\
\text { Schüler*innenschaft. }\end{array}$ & $\begin{array}{l}\text { - Inklusive Schule 1-13. } \\
\text { - Heterogenität der Schule repräsentativ für die Bevölkerung. } \\
\text { - } \text { Mehr Chancen durch internationale Standards der Anti-Diskriminie- } \\
\text { rung, der Berücksichtigung der Lernvoraussetzungen auch nach } \\
\text { benachteiligenden Faktoren und entsprechende Förderpläne nach } \\
\text { individuellem Bedarf für alle. } \\
\text { - Demokratie im Kleinen in der Lernlandschaft durch umfassende } \\
\text { Beteiligungsmöglichkeiten, Mitbestimmung bei Beziehungen und } \\
\text { Inhalten. } \\
\text { - Programme und Hilfen für Menschen, die noch nicht hinreichend } \\
\text { Deutsch sprechen. } \\
\text { - Ein klares und nachhaltiges Konzept gegen Mobbing. } \\
\text { - Ein klares und nachhaltiges Konzept, um Eltern, Familien, die } \\
\text { Nachbarschaft und das Viertel/die Stadt an Schulprozessen zu } \\
\text { beteiligen. }\end{array}$ \\
\hline
\end{tabular}




\begin{tabular}{|c|c|c|}
\hline $\begin{array}{c}\text { Merk- } \\
\text { mal }\end{array}$ & Leitprinzipien & Beispiele konkreter Maßnahmen \\
\hline 3 & $\begin{array}{l}\text { Eine Schule mit hohen } \\
\text { Qualifikationsstandards } \\
\text { für alle Lernenden, } \\
\text { die möglichst hohe } \\
\text { Abschlüsse für alle } \\
\text { erreichen will und mehr } \\
\text { Lernende zum Abitur } \\
\text { führen soll als der } \\
\text { Durchschnitt deutscher } \\
\text { Schulen. }\end{array}$ & $\begin{array}{l}\text { - Ein durchgehend kompetenzorientierter Ansatz mit entsprechender } \\
\text { Struktur und Hilfen: Kompetenzraster, Feedback, Ziel- und Förder- } \\
\text { gespräche, umfassendes digitales Lernen. } \\
\text { - Viele Lernzugänge, Lernperspektiven und Ergebnisse durch Anpas- } \\
\text { sung des Curriculums an Lernbedürfnisse (Curriculumwerkstätten } \\
\text { der Teams helfen, dies kreativ zu erreichen), Mischung von Selbst- } \\
\text { lern-, Projekt- und Wahlthemen in größeren Zeitfenstern. } \\
\text { - Lernformate (Selbstlernphasen, Projekte, Werkstätten) sichern } \\
\text { eine gute Mischung aus eigenständigem und sozialem Lernen, aus } \\
\text { Vorgaben und eigenen Interessen ab. } \\
\text { - Erstellung eines auf die Lernenden hinreichend abgestimmten } \\
\text { Selbstlernmaterials in Hauptfächern. } \\
\text { - Sorge für Basisqualifikationen bei allen, Ermöglichung von Niveau- } \\
\text { stufen und Hilfen für alle Lernherausforderungen. } \\
\text { - Mischung aus verpflichtenden Inhalten zum Bestehen zentraler } \\
\text { Prüfungen und Freiräumen für Interessen und Neigungen. } \\
\text { - Differenzierung nach den unterschiedlichen Lernbedürfnissen von } \\
\text { der Standortbestimmung aller bis hin zu individuellen Förderplänen. } \\
\text { - Durchgehendes Feedback, Evaluation und Supervision bei Lernfra- } \\
\text { gen, um persönliche Exzellenz je nach Möglichkeiten zu erreichen. } \\
\text { - Die Schularchitektur unterstützt durch die Gestaltung des Gebäu- } \\
\text { des die pädagogischen Intentionen insbesondere durch flexible und } \\
\text { mobile Möblierungen in offenen und leicht veränderbaren Lernland- } \\
\text { schaften. }\end{array}$ \\
\hline 4 & $\begin{array}{l}\text { Eine durchgehend } \\
\text { gebundene Ganztags- } \\
\text { schule mit einer guten } \\
\text { Rhythmisierung von } \\
\text { Arbeits- und Entspan- } \\
\text { nungsphasen, von } \\
\text { Eigenzeiten und sozia- } \\
\text { len Zeiten verteilt über } \\
\text { den ganzen Tag. }\end{array}$ & $\begin{array}{l}\text { - Gleitzeit für Ankunft und Schluss. } \\
\text { - Aktives und interaktives Lernen als Grundregel, Instruktionen nur } \\
\text { noch soweit nötig und in die Lernformate integriert. } \\
\text { - Je etwa } 30 \text { Prozent für Selbstlernzeiten, Projekte und Werkstätten; } \\
\text { Aufteilung der meisten Schulfächer in diese Formate; Werkstätten } \\
\text { können Spezialgebiete aus den Schulfächern fokussieren (z. B. statt } \\
\text { Sport Tischtennis usw.). } \\
\text { - Werkstätten sollen ein gleiches Zeitfenster für alle Jahrgänge ha- } \\
\text { ben, um über die Jahrgänge hinweg Angebote machen zu können } \\
\text { (z. B. Schach von } 1 \text { bis 13). } \\
\text { - Abschaffung des 45-Minuten-Taktes zugunsten größerer Zeitfenster } \\
\text { in den Plänen der jeweiligen Lernlandschaften. } \\
\text { - Leichter Zugang zu Außenterrassen nach Bedarf. } \\
\text { - Zugang zu einem großen Bewegungsraum, der gleichzeitig als } \\
\text { kleine Aula fungiert. } \\
\text { - Große Mensa, gut ausgestattete Bibliothek mit Medien, gro- } \\
\text { ße Aufenthalts- und Ausstellungsflächen, Rückzugsbereiche, } \\
\text { Schüler*innenfirmen, Fachräume. }\end{array}$ \\
\hline
\end{tabular}


Die „Heliosschule - Inklusive Universitätsschule der Stadt Köln“ |

\begin{tabular}{|c|c|c|}
\hline $\begin{array}{c}\text { Merk- } \\
\text { mal }\end{array}$ & Leitprinzipien & Beispiele konkreter Maßnahmen \\
\hline 5 & $\begin{array}{l}\text { Eine anregende } \\
\text { und unterstützende } \\
\text { Lernumgebung, die } \\
\text { unterschiedliche Förde- } \\
\text { rungen für das Lernen } \\
\text { und Erfahrungen im } \\
\text { Blick auf unterschied- } \\
\text { liche Bedürfnisse und } \\
\text { Interessen der Lernen- } \\
\text { den (jede*r ist anders) } \\
\text { ermöglicht. }\end{array}$ & $\begin{array}{l}\text { - Individualisierung des Lernens durch Lernformate, wobei auf Lern- } \\
\text { voraussetzungen in umfassender Weise Rücksicht nach Vorkennt- } \\
\text { nissen, Lernzugängen, Lernwegen, Lernmöglichkeiten und begleite- } \\
\text { ten Lernfortschritten genommen wird. } \\
\text { - Konstruktivistischer Lernansatz, der Konstruktion gegenüber der } \\
\text { Instruktion bevorzugt und folgende Aspekte besonders im Curricu- } \\
\text { lum beachtet: } \\
\text { - Multiperspektivität, um unterschiedliche Perspektiven zu ermögli- } \\
\text { chen und vielfältige Sichtweisen einzubringen; } \\
\text { - Multimodalität, um unterschiedliche Lernzugänge für unterschied- } \\
\text { liche Lernende zu ermöglichen; } \\
\text { - Multiproduktivität, um unterschiedliche Lernergebnisse in hoher } \\
\text { Anzahl zu erzielen. } \\
\text { - Ein Netzwerkansatz und digitales Lernen helfen der Schule, um } \\
\text { eine Vielfalt von Medien in einem aktiven und konstruktiven Ge- } \\
\text { brauch zu nutzen; dabei kann zugleich eine medienkritische Einstel- } \\
\text { lung vermittelt werden. }\end{array}$ \\
\hline 6 & $\begin{array}{l}\text { Die Schule sieht alle } \\
\text { Lernenden als einzigar- } \\
\text { tig an, und alle haben } \\
\text { besondere Bedürfnisse } \\
\text { und Interessen, die } \\
\text { jeweils Chancen zur } \\
\text { Verwirklichung und } \\
\text { Förderung benötigen. }\end{array}$ & $\begin{array}{l}\text { - Standortbestimmung in der Lerndiagnostik für alle, besondere } \\
\text { Diagnostik bei bestimmten Förderbedarfen. } \\
\text { - Zielvereinbarungen mit Fördergesprächen und Feedback, um Lern- } \\
\text { fortschritte zu ermöglichen und niemanden zurückzulassen. } \\
\text { - Ermöglichung persönlicher Exzellenz für alle als grundlegendes } \\
\text { Leitziel (Good-Work-Ansatz). } \\
\text { - Eine hinreichende Anzahl von Sonderschullehrkräften und weiterem } \\
\text { spezialisiertem Personal, um die Breite der Lernförderungen abzu- } \\
\text { decken. } \\
\text { - Hinzuziehung von weiterer externer Expertise bei Bedarf. } \\
\text { - Vorgehen nach den Kriterien der WHO (ICF). }\end{array}$ \\
\hline 7 & $\begin{array}{l}\text { Ein komplexer Ansatz } \\
\text { der Beurteilung, um den } \\
\text { Lernenden ein zeitna- } \\
\text { hes Feedback zu geben } \\
\text { und ihre Lernfortschritte } \\
\text { umfassend und stüt- } \\
\text { zend zu begleiten. }\end{array}$ & $\begin{array}{l}\text { - Feedback sowohl für Fortschritte im individuellen als auch im sozi- } \\
\text { alen Lernen. } \\
\text { - Beurteilungen nicht nur im Blick auf Leistungen und Verhalten, } \\
\text { sondern auch für das Wachstum in den Lernprozessen und den } \\
\text { Zugewinn an Kompetenzen. } \\
\text { - Ein systemisches Modell der Beurteilung, das Inhalte und Bezie- } \\
\text { hungen in ihrer Wechselwirkung beachtet. } \\
\text { - Eine transparente Beurteilung, die verbale Formen gegenüber } \\
\text { vereinfachten Notensystemen bevorzugt. } \\
\text { - Eine Beurteilung, die nah an den Lernaufgaben und Ergebnissen } \\
\text { wie Verhaltensweisen ansetzt, nach Lernstufen differenziert, Lern- } \\
\text { fortschritte für halbjährliche Gespräche mit Lernenden und Eltern } \\
\text { dokumentiert. } \\
\text { - Feedup, Feedback, Feedforward als notwendige Bestandteile der } \\
\text { kontinuierlichen Lernbegleitung. } \\
\text { - Insgesamt eine Mischung aus einer Diagnose von Startpunkten, } \\
\text { kontinuierlichem Feedback und dessen Dokumentation, Förderge- } \\
\text { sprächen bei Bedarf, halbjährlichen Beurteilungs- und neuen Ziel- } \\
\text { vereinbarungsgesprächen. }\end{array}$ \\
\hline
\end{tabular}




\begin{tabular}{|c|c|c|}
\hline $\begin{array}{c}\text { Merk- } \\
\text { mal }\end{array}$ & Leitprinzipien & Beispiele konkreter Maßnahmen \\
\hline 8 & $\begin{array}{l}\text { Die Schularchitektur } \\
\text { löst die Klassenräume } \\
\text { auf und ermöglicht } \\
\text { Lernlandschaften, in } \\
\text { denen in neuen Zeit- } \\
\text { strukturen eine enge } \\
\text { Verbindung des multi- } \\
\text { professionellen Teams } \\
\text { mit den Lernenden } \\
\text { gelingt, wobei eine } \\
\text { anregende, förderliche } \\
\text { und sichere sowie } \\
\text { wohnliche Atmosphäre } \\
\text { in Lernzonen unter- } \\
\text { schiedlichen Lernbe- } \\
\text { dürfnissen entspricht. }\end{array}$ & $\begin{array}{l}\text { - Neubau, der umfassend zwischen Architekten und Nutzer*innen in } \\
\text { einer Phase Null geplant wurde (Montag Stiftungen, 2017). } \\
\text { - } \text { Barrierefreier Bau, bei dem besonders Licht, Akustik, Zugänge, } \\
\text { Fluchtwege, Außenraum und viele Details einer guten Lernumge- } \\
\text { bung beachtet sind. } \\
\text { - } \text { Multifunktional veränderbare Lernlandschaften; sie enthalten Zo- } \\
\text { nierungen, die in Rotation von den Stammgruppen genutzt werden } \\
\text { können, aber auch durch die gesamte Lerngruppe in Selbstlernpha- } \\
\text { sen. } \\
\text { - Die Zonen enthalten z. B. Flächen für stilles Arbeiten und } \\
\text { Präsentieren, Loungebereich, kleines Auditorium, abgeschlossene } \\
\text { Räume, Thinktank, kreisförmige Versammlung von Stammgruppen, } \\
\text { Aula für alle, Küchenzeile, Waschräume und Toiletten, Garderobe, } \\
\text { Außenterrassen. } \\
\text { - Die kleine Aula kann jeweils von zwei Lernlandschaften genutzt } \\
\text { werden; sie ist zugleich ein Bewegungsraum oder Hörsaal; sie kann } \\
\text { räumlich vielfältig durch Abtrennungen für Ausstellungen, verschie- } \\
\text { dene Groß- oder Kleingruppenmethoden genutzt werden. } \\
\text { - Zusätzliche Räume stehen als weiteres Angebot zur Differenzierung } \\
\text { zur Verfügung (z. B. für Sport, Musik, Kunst, Werkstätten, Bibliothek } \\
\text { usw.). }\end{array}$ \\
\hline 9 & $\begin{array}{l}\text { Die Schule öffnet sich } \\
\text { vielfältig in die Lebens- } \\
\text { welt. }\end{array}$ & $\begin{array}{l}\text { - Die Lebenswelt soll sowohl in die Schule kommen als auch von } \\
\text { innen nach außen gelebt werden (Theater, Café, Projekte usw.). } \\
\text { - Schüler*innenfirmen helfen, Lebenswelt- und Berufsbezüge herzu- } \\
\text { stellen; sie haben Außenzugänge zur Straße. } \\
\text { - Studierende werden aktiv in alle Schulprojekte einbezogen. } \\
\text { - Die Schule öffnet sich für Nachbarschaft, Viertel und Stadt in vielen } \\
\text { Aktivitäten und Dienstleistungen; sie bildet Netzwerke und Verein- } \\
\text { barungen mit Geschäften und sozialen Organisationen. } \\
\text { - Es werden enge Kontakte zur Berufswelt gepflegt, um } \\
\text { Berufseinsteiger*innen ohne Abitur Zugänge zu erleichtern. }\end{array}$ \\
\hline 10 & $\begin{array}{l}\text { Evaluation, Coaching } \\
\text { und Supervision sichern } \\
\text { eine kritische Beurtei- } \\
\text { lung der Fortschritte der } \\
\text { Schule in allen Berei- } \\
\text { chen. }\end{array}$ & $\begin{array}{l}\text { - Kollegiale Beratung ist eine Regel in den multiprofessionellen } \\
\text { Teams. } \\
\text { - Außenevaluation ist Teil der wissenschaftlichen Begleitung durch } \\
\text { die Universität, und die Ergebnisse werden kontinuierlich veröffent- } \\
\text { licht. } \\
\text { - Coaching und Supervision werden sowohl vom schulpsychologi- } \\
\text { schen Dienst als auch durch Unterstützung der Montag Gesell- } \\
\text { schaft und der Universität wahrgenommen. } \\
\text { - Expertise von außen wird insbesondere bei Fragen spezieller För- } \\
\text { derbedarfe eingeholt; vielfach verfügt die Universität über diese } \\
\text { Expertise. } \\
\text { - Die Universität evaluiert zudem die Lehramtsausbildung in der } \\
\text { Schule und zieht hieraus Schlüsse für eine Verbesserung der Aus- } \\
\text { bildung insgesamt. }\end{array}$ \\
\hline
\end{tabular}

\section{Ausblick}

Die „Heliosschule“ könnte viele ihrer Vorstellungen leichter realisieren, wenn sie eine Privatschule wäre, weil dann die engen und hinderlichen Vorgaben u.a. im Bereich Eine Schule für alle (Klassen 1-13), in der Umstellung der Beurteilung von Noten auf verbale Beurteilungen und in einer flexibleren Verwendung von Geldern besei- 
tigt werden könnten. Obwohl insbesondere diese Vorteile in der Konzipierung der Schule sehr gewünscht waren, so sollte die Schule dennoch trotz solcher Hindernisse öffentlich sein, denn es geht diesem Konzept darum zu zeigen, wie eine inklusive Regelschule auch innerhalb eines schulrechtlichen Rahmens funktionieren kann, der noch keineswegs hinreichend auf die schulischen Bedürfnisse für eine gute Schulentwicklung und insbesondere nicht für Inklusion ausgelegt ist. Bei der derzeitigen zögerlichen Umsetzung der Inklusion auch in NRW, bei der gegenwärtig auf inklusive Schwerpunktschulen gesetzt wird, besteht die Gefahr, dass eine Heterogenität, die repräsentativ für die Bevölkerung ist, für die verbleibenden inklusiven Schulen kaum mehr gewährleistet werden kann. Für die „Heliosschule“ wird dies dann zum Problem, wenn sie überproportional Lernende mit Förderbedarf versorgen muss, so dass die Chancen der Heterogenität einer guten Mischung von Schüler*innen sich in Risiken einer Belastung durch zu viele Schüler*innen mit Förderbedarfen verwandeln. Eine solche Politik verstößt zwar klar gegen die UN-Behindertenrechtskonvention, der Deutschland beigetreten ist, aber die Bildungspolitik steht noch sehr im Bann traditioneller, segregierender Vorstellungen. Eine inklusive Schule - dies ist aus der Sicht der internationalen Forschung und Politik zu folgern - muss eine heterogene Regelschule für alle sein, wenn sie überhaupt erfolgreich sein soll.

Vor diesem Hintergrund gibt es vor allem drei Risiken in der praktischen Umsetzung der Ziele der „Heliosschule“:

1. Inwieweit gelingt die Aufnahme einer heterogenen Schüler*innenschaft in der ohnehin bereits durch viele soziale Probleme charakterisierten Bevölkerung der Stadt Köln? Je mehr sich inklusive Schwerpunktschulen in neue Förderschulen verwandeln, desto weniger können die Ansprüche, die die UN an einen inklusiven Regelschulbesuch stellt, erfüllt werden. Dies gilt insbesondere auch für die personelle und sachliche Ausstattung, die sich in Deutschland deutlich verbessern müsste. In anderen Ländern, die erfolgreicher in der Inklusion sind, geht man längst von einer Zusatzausstattung von 20 Prozent als Quote über alle Schüler*innen aus, um eine hinreichend qualitätsvolle Schule für alle zu entwickeln. Inklusion betrifft eben nicht nur Menschen mit Behinderungen, sondern auch alle Gruppen von Benachteiligungen durch Armut, Migration, Diskriminierungen. Sie betrifft aber auch Menschen mit besonderen Begabungen, die im herkömmlichen Schulsystem nicht hinreichend gefördert werden.

2. Inwieweit gelingt es, das bestehende und noch auf die Exklusion ausgelegte Schulrecht zu erweitern und zu verändern, um insbesondere im Bereich der neuen Lernformate, Beurteilungen und Standortbestimmungen so zu arbeiten, dass ein breites Anforderungsprofil einer inklusiven Schule möglich ist? Inklusion ist zwar wesentlich eine Frage der Haltung der Lehrkräfte und des multiprofessionellen Teams, aber sie gelingt nur dann, wenn auch der bürokratische Rahmen solche Haltungen nicht verhindert oder erschwert. Erfolgreiche inklusive Länder zei- 
gen, dass hier die einzelnen Schulen deutlich größere Freiheiten als die deutschen Schulen haben.

3. Schließlich ist es eine wichtige Frage, inwieweit es das multiprofessionelle Team trotz der gegebenen Hindernisse schaffen kann, die Inklusion in einem Modell konkret umzusetzen, ohne sich an den Hindernissen aufzureiben oder zu große Reibungsverluste zu erleiden.

Für diese und andere Fragen wird es interessant sein, welche wissenschaftlichen Ergebnisse die Begleitung durch die Universität zu Köln erheben wird, wenn dieses Experiment in seiner weiteren Entwicklung analysiert wird. Die Eckvereinbarungen dazu sind zunächst günstig:

- Die Universität hat sich sowohl durch eine hohe Investition von Geldern in die Gebäude als auch die Ausstattung des Lehrprofils selbst verpflichtet, in diesem Modell das Zusammenwirken von sehr guter inklusiver Praxis mit einer gezielt vorbereiteten Lehramtsausbildung zu untersuchen. Dadurch, dass dies in Abstimmung mit der Schule geschieht, lassen sich Untersuchungen führen, die in beiderseitigem Interesse liegen.

- Aus Sicht der Schule sind zahlreiche spezielle Forschungsfragen relevant, die sich aus dem Bedarf der Praxis ergeben werden und an die Forschung herangetragen werden können. Dies kann den Praxisbezug der Forschung erhöhen.

- Dabei kann zugleich in Forschungen zu Langzeitwirkungen erhoben werden, inwieweit die international bereits als erfolgreich geltenden Strategien inklusiven Lehrens und Lernens auch an einer deutschen Schule umsetzbar sind und zu welchen Ergebnissen dies führt.

- Auch Vergleiche des Modells der Lernformate mit herkömmlichen Schulen sind von großem Interesse. Wenn sich der Einsatz der neuen Lernorganisation durch Fortschritte in den Leistungen bewährt, wie es auch schon einige Beispiele zeigen (vgl. Reich, 2017), dann hätte dies auch Konsequenzen für Reformen im Schulsystem.

- Schließlich kann sich genauer zeigen, inwieweit die doch recht kurze Praxisphase der Studierenden von knapp einem halben Jahr ausreicht, um ihnen in einem BestPractice-Modell hinreichend Erfahrungen und Kompetenzen zukommen zu lassen, die sie in einem späteren Einsatz an anderen Schulen dann nutzen könnten.

\section{Literatur und Internetquellen}

Dewey, J. (1882-1924). Collected Works. Edited by J.A. Boydston. The Early Works (EW 1-5): 1882-1898. Carbondale \& Edwardsville, IL: Southern Illinois University Press / London \& Amsterdam: Feffer \& Simons; The Middle Works (MW 1-15): 1899-1924. Carbondale \& Edwardsville, IL: Southern Illinois University Press. 
Garrison, J., Neubert, S., \& Reich, K. (2012). John Dewey's Philosophy of Education. An Introduction and Recontextualization for Our Times. New York: Palgrave Macmillan. doi.org/10.1057/9781137026187

Garrison, J., Neubert, S., \& Reich, K. (2016). Democracy and Education Reconsidered. 100 Years after Dewey. London \& New York: Routledge.

Kricke, M., \& Reich, K. (2016). Teamteaching. Weinheim: Beltz.

Kricke, M., Schanz, L., Reich, K., \& Schneider, J. (2018). Raum und Inklusion. Weinheim: Beltz.

Montag Stiftungen (Hrsg.). (2017). Schulen planen und bauen 2.0 - Grundlagen, Prozesse, Projekte. Berlin \& Seelze: Jovis \& Friedrich.

Reich, K. (2012). Inklusion und Bildungsgerechtigkeit. Weinheim: Beltz.

Reich, K. (2014). Inklusive Didaktik. Weinheim: Beltz.

Reich, K. (Hrsg.). (2017). Inklusive Didaktik in der Praxis. Beispiele erfolgreicher Schulen. Weinheim et al.: Beltz.

Reich, K. (2018). The Helios School - Inclusive University School in the City of Cologne. International Research in Higher Education, 3 (1). doi.org/10.5430/irhe.v3n1p4

Reich, K., Asselhoven, D., \& Kargl, S. (2015). Eine inklusive Schule für alle - Das Modell der Inklusiven Universitätsschule. Weinheim: Beltz.

Kersten Reich, Univ.-Prof. em. Dr., Tätigkeit im Forschungsgebiet „Internationale Lehr- und Lernforschung“ an der Universität zu Köln mit den Hauptarbeitsgebieten Konstruktivismus und Kulturtheorien, konstruktivistische und systemische Pädagogik und Didaktik; wissenschaftlicher Leiter der Gründung der „Heliosschule - Inklusive Universitätsschule der Stadt Köln“.

E-Mail: Kersten.Reich@uni-koeln.de 